\title{
Observing a Unicycle Robot with Data Rate Constraints: a Case Study
}

\author{
Quentin Voortman, Denis Efimov, Alexander Pogromsky, Haik Silm, Jean-Pierre Richard, and Henk Nijmeijer
}

\begin{abstract}
In this paper, we consider the problem of remote observation of a unicycle-type mobile robot through a data rate constrained communication channel, which can only send a limited number of bits per unit of time. The objective is to reconstruct estimates of the state of the robot at the remote location through the messages that are sent. The design of the communication protocol should ensure that the maximum observation error is bounded whilst using as few bits per unit of time as possible. An event-triggered observation scheme is developed specifically for the unicycle-type robot. This observer is tested through experiments on Turtlebots. The experiments show that the event-triggered scheme is very efficient at reducing the average number of required communications.
\end{abstract}

\section{INTRODUCTION}

As wireless communication technologies have become omnipresent in modern society, the world of dynamics and control has been taken over by them as well. There are many different applications where one or several dynamical systems or the components thereof are connected via data rate constrained communication channels. Examples include: cooperative load displacement by robots, underwater communication of autonomous vehicles, formation control for drones, cooperative cruise control, etc. All these problems share the common feature that some source of uncertainty (in the sense of [22]) makes it necessary to communicate over a communication channel that is limited either in the transmission rate of packets, the size of packets, or both. The sources of uncertainty include perturbations, noise, parametric uncertainty, and sensitivity to initial conditions. Although the problem of communication can be seen separately from the control/observation associated with the underlying dynamical systems, a combined approach allows one to provide better results, be it in terms of control performance, error bounds, sufficient rates, etc.

Among the many works in this field, we note the pioneering contributions [29], [5] which provide results for linear systems. Many other results were obtained for linear systems and broad overviews of such results can be found in [4], [9], [2]. For nonlinear systems, early important results include [21] and [15], which use the concept of entropy to characterize the minimum data rates. Many more papers exploited entropy-based techniques to provide constructive bounds on the sufficient/necessary data rates (see [12], [19], [13], [23], [16], [17], [24], [28] and [18]).

At the same time as data rate constrained control appeared in the literature, another topic emerged: event-triggered control. Two of the earliest works in this field include [1] and [3]. An introduction

Quentin Voortman, Alexander Yu. Pogromsky and Henk Nijmeijer are with the Department of Mechanical Engineering, Eindhoven University of Technology, Eindhoven, The Netherlands. Alexander Yu. Pogromsky is also with the Department of Control Systems and Robotics, Saint-Petersburg National Research University of Information Technologies, Mechanics, and Optics (ITMO), St. Petersburg, Russia. Quentin Voortman, together with Denis Efimov and Jean-Pierre Richard is with Inria, University of Lille, CNRS, UMR 9189-CRIStAL, Centrale Lille F-59000 Lille, France. Haik Silm is with the Department of Computer Science, KU Leuven, Celestijnenlaan 200A, 3001 Leuven, Belgium. \{q.j.t.voortman@tue.nl\}.

This paper was elaborated in the UCoCoS project which has received funding from the European Union's Horizon 2020 research and innovation programme under the Marie Skłodowska-Curie grant agreement No 675080. to event-based control can be found in [8] and an overview of sampling-related results in [10]. Event-triggered control and data rate constrained control have recently been combined in some works of the literature. Examples of such works include: [7] (eventtriggered sensor schedule for remote estimation for a linear system), [25] (distributed state estimation with data rate constraints), [30] (networked state estimation with a shared communication medium) and, [20] (LMI approach is used for the networked state estimation problem over a shared communication medium).

In this paper, we present the theory behind a data rate constrained observer for a unicycle robot, which is modeled by a nonlinear dynamical system and has already been studied extensively (see e.g. [11], [6] or [14]). The robot is equipped with a smart sensor, capable of measuring the position and orientation of the robot and performing computations. It is connected to a remote location via a communication channel. The smart sensor can send messages over this communication channel to the remote location to provide an estimate of the position of the robot at the remote location. The particularity of the communication channel is that it is restricted in terms of data rates that it can transmit. The objective is to develop a communication protocol such that it is possible to reconstruct the position of the robot at a remote location whilst using limited data rates. The novelty of the result and the main contribution of this work consists of using an event-triggered communication protocol which often greatly reduces the communication rate, as is proven through experiments on mobile robots. To the best of the authors' knowledge, no fundamental bounds on the minimum sufficient capacity to observe unicycle-type robots have been obtained in the literature.

\section{Problem Statement}

We consider a unicycle-type robot with the following dynamics:

$$
\left[\begin{array}{c}
\dot{x}_{1}(t) \\
\dot{x}_{2}(t) \\
\dot{\theta}(t)
\end{array}\right]=\left[\begin{array}{c}
\left(u_{x}(t)+d_{x}(t)\right) \cos (\theta(t)) \\
\left(u_{x}(t)+d_{x}(t)\right) \sin (\theta(t)) \\
u_{\theta}(t)+d_{\theta}(t)
\end{array}\right], \quad \forall t \geq 0,
$$

where $x=\left(x_{1}, x_{2}\right) \in \mathbb{R}^{2}$ is the position of the robot, $\theta \in S^{1}$ is the orientation angle of the robot, $u=\left(u_{x}, u_{\theta}\right) \in \mathbb{R}^{2}$ is the input, $d_{x} \in$ $\mathbb{R}$ and $d_{\theta} \in \mathbb{R}$ are time-varying input perturbations. The system's output $y$ consists of the full state, sampled with sampling interval $\bar{t}$,

$$
y\left(t_{k}\right)=\left[\begin{array}{c}
x_{1}\left(t_{k}\right) \\
x_{2}\left(t_{k}\right) \\
\theta\left(t_{k}\right)
\end{array}\right], \quad \forall k \geq 0,
$$

where $t_{k}=\bar{t} k$ are the sampling instants. We assume that the input perturbations are continuous signals that verify

$$
\left|d_{i}(t)\right| \leq \delta_{i},
$$

$\forall i \in\{x, \theta\}, \forall t \geq 0$ where $\delta_{i}$ are the maximum input perturbations, which are known constants.

The system is connected to a remote location via a data rate constrained communication channel. Several devices interact to send messages $m\left(s_{j}\right)$ (where $s_{j}$ are the transmission times) over 
this communication channel. These devices are a smart sensor (a sensor admitting some computational capacities, which allows it to perform additional computations on the measured data), an alphabet function $\mathscr{A}$, and a decoder $\mathscr{D}$. The smart sensor is further subdivided into a sampler $\mathscr{S}$ and a coder $\mathscr{C}$. Together, the devices form a communication protocol. The smart sensor sends messages over the communication channel to the decoder which interprets the messages to generate an estimate $\hat{x}(t)$ of the position $x(t)$ of the robot. Fig. 1 depicts how the different components interact. Note that only an estimate of the position should be generated at the remote location.

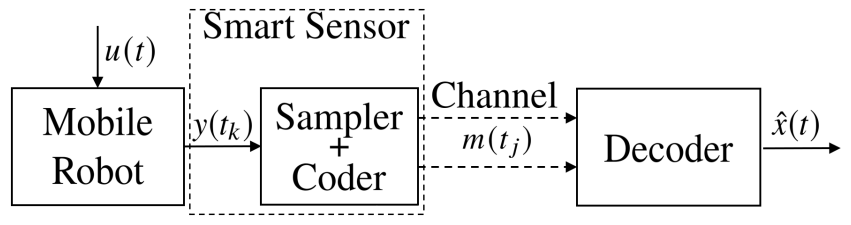

Fig. 1. Structure of the interactions between the different components of the problem.

The sensor and the decoder share the common knowledge of an initial estimate $\hat{x}_{0}$ which satisfies

$$
\left\|x(0)-\hat{x}_{0}\right\|_{2} \leq \varepsilon_{0},
$$

where $\varepsilon_{0}$ is a user-specified parameter corresponding to the error in initial conditions and $\|\cdot\|_{2}$ is to the Euclidean norm in $\mathbb{R}^{n}$. We define the following metric on $S^{1}$

$$
d_{S}\left(\theta_{1}, \theta_{2}\right):=\left\{\begin{array}{cc}
\left|\theta_{1}-\theta_{2}\right|, & \text { if }\left|\theta_{1}-\theta_{2}\right| \leq \pi \\
2 \pi-\left|\theta_{1}-\theta_{2}\right|, & \text { else. }
\end{array}\right.
$$

The sensor and remote location also have an initial estimate $\hat{\theta}_{0}$, which satisfies

$$
d_{S}\left(\theta(0), \hat{\theta}_{0}\right) \leq \varepsilon_{\theta_{0}} .
$$

The knowledge of the following quantities is shared by all devices: the maximum input perturbations $\delta_{x}$ and $\delta_{\theta}$, the discretization errors $\varepsilon_{x}$ and $\varepsilon_{\theta}$ (which are induced by coding/decoding operation and for brevity of exposition in this document the constants $\varepsilon_{0}=\varepsilon_{x}$ and $\varepsilon_{\theta_{0}}=\varepsilon_{\theta}$ ), and the initial estimates $\hat{x}_{0}$ and $\hat{\theta}_{0}$.

At the system side, the sampler $\mathscr{S}$ generates the instants of transmission in the following way

$$
s_{j+1}=\mathscr{S}\left(s_{j},\left\{y\left(t_{k}\right)\right\}_{k: t>t_{k} \geq 0}, m\left(s_{1}\right), \ldots, m\left(s_{j}\right)\right),
$$

$s_{0}=0, m(0)=\emptyset$, with the restriction that $s_{j+1}>s_{j}$. The coder then generates the messages in the following way

$$
m\left(s_{j}\right)=\mathscr{C}\left(\left\{y\left(t_{k}\right)\right\}_{k: s_{j}>t_{k} \geq 0}, m\left(s_{1}\right), \ldots, m\left(s_{j-1}\right)\right),
$$

$\forall s_{j}: j>0$. At each communication instant, the list of different possible messages is encoded into a finite-sized alphabet (the finite cardinality is necessary because of the data rate constraints). The alphabet function $\mathscr{A}$ determines the last index of the messages $l_{j}$ in the following way

$$
l_{j}=\mathscr{A}\left(m\left(s_{1}\right), \ldots, m\left(s_{j}\right)\right), \quad \forall s_{j}: j>0 .
$$

The restriction on the choice of messages is $m\left(s_{j}\right) \in\left\{1, \ldots, l_{j}\right\}$, $\forall s_{j}: j>0$. At the remote location, the decoder $\mathscr{D}$ receives the messages and interprets them to generate an estimate of the state $\hat{x}(t)$ in the following way

$$
\hat{x}(t)=\mathscr{D}\left(m\left(s_{1}\right), \ldots, m\left(s_{j}\right)\right), \quad \forall t \in\left[s_{j}, s_{j+1}\right),
$$

$\forall j \geq 0$. The number of bits $b_{j}$ required to encode the messages depends on the length of the alphabet. In practice, this implies that

$$
b_{j}:=\left\lceil\log _{2} l_{j}\right\rceil \quad \forall s_{j}: j>0,
$$

where $\lceil\cdot\rceil$ is the ceiling function (which rounds any real number to the smallest integer larger than or equal to that number). The communication rate $R$ resulting from the transmission of these messages is defined as

$$
R:=\lim _{j \rightarrow \infty} \frac{1}{s_{j}} \sum_{i=1}^{j} b_{i} .
$$

The rate is thus defined such that it is the average overall communication instants of the number of bits that are sent. Because of the perturbation, measurement error, and finite communication rate, it is impossible to generate estimates of the state at the remote location with zero error. Instead, the design of the communication protocol should ensure that the maximum observation error $\eta$ is bounded, where

$$
\eta:=\sup _{t \geq 0}\|x(t)-\hat{x}(t)\|_{2}
$$

Note that the maximum observation error only concerns the position of the robot and not its angular orientation. The first objective of this paper is to design a communication protocol, in the form of a Sampler $\mathscr{S}$, Coder $\mathscr{C}$, Alphabet $\mathscr{A}$ and Decoder $\mathscr{D}$ such that the maximum observation error and the rate are bounded, and preferably as small as possible. The second objective is to test this communication protocol through experiments on mobile robots.

\section{DESIGNING THE OBSERVER}

In this section, we introduce the different agents of the communication protocol. Before we describe the communication protocol and due to page number limitations, we pose the following simplifying assumption about the input.

Assumption 1: The inputs $u_{x}(t)$ and $u_{\theta}(t)$ are constant, i.e. $u_{x}(t)=\bar{u}_{x}, u_{\theta}(t)=\bar{u}_{\theta}, \forall t \geq 0$ and known.

Remark 1: This hypothesis can be relaxed to the requirement that the inputs are piece-wise constant keeping their values over an interval of time, in which case the input values have to be also communicated. For more information about how to transmit estimates of the input, one can refer to [27].

We also introduce the system

$$
\left[\begin{array}{c}
\dot{\hat{x}}_{1}(t) \\
\dot{\hat{x}}_{2}(t) \\
\dot{\hat{\theta}}(t)
\end{array}\right]=\left[\begin{array}{c}
\bar{u}_{x} \cos (\hat{\theta}(t)) \\
\bar{u}_{x} \sin (\hat{\theta}(t)) \\
\bar{u}_{\theta}
\end{array}\right]
$$

which corresponds to (1) with zero perturbations. Note that for any initial condition $\left[\begin{array}{lll}\hat{x}_{10} & \hat{x}_{20} & \hat{\theta}_{0}\end{array}\right]^{\top},(12)$ has the following exact solution

$$
\begin{aligned}
\hat{x}_{1}(t) & =\frac{\bar{u}_{x}}{\bar{u}_{\theta}}\left(\sin \left(\bar{u}_{\theta} t+\hat{\theta}_{0}\right)-\sin \left(\hat{\theta}_{0}\right)\right)+\hat{x}_{10}, \\
\hat{x}_{2}(t) & =\frac{\bar{u}_{x}}{\bar{u}_{\theta}}\left(-\cos \left(\bar{u}_{\theta} t+\hat{\theta}_{0}\right)+\cos \left(\hat{\theta}_{0}\right)\right)+\hat{x}_{20}, \\
\hat{\theta}(t) & =\bar{u}_{\theta} t+\hat{\theta}_{0} .
\end{aligned}
$$

The main mechanism of the communication protocol can be described as follows: at the sensor side, the sampled state $y\left(t_{k}\right)$ is measured at sampling instants $t_{k}$. The smart sensor simulates a virtual copy of the decoder, which is possible since the sensor generates the messages that the decoder receives. The sensor is thus aware of a copy of the remote estimate, which will be denoted $\hat{x}_{c}(t)$. The sensor sends messages which contain information necessary to reconstruct both $\hat{x}\left(s_{j}\right)$, as well as $\hat{\theta}\left(s_{j}\right)$. Starting at the estimates 
stemming from the last message and in the absence of messages, the decoder simply updates the estimate by computing the solution of (12) with $\hat{x}\left(s_{j}\right)$ and $\hat{\theta}\left(s_{j}\right)$ as an initial condition. If at some sampling instant $t_{k}$, the distance between $y\left(t_{k}\right)$ and $\hat{x}_{c}\left(t_{k}\right)=\hat{x}\left(t_{k}\right)$ becomes larger than some prescribed maximum error, the sampler decides to communicate: it sets $s_{j}=t_{k}$ and the coder then sends a message to the decoder to provide new estimates $\hat{x}\left(t_{j}\right)$ and $\hat{\theta}\left(t_{j}\right)$.

The communication procedure, which we will further reference as Procedure 1, is composed of a sampler, alphabet, coder, and decoder as described below. Although the problem statement leaves room for different choices of communication instants, our communication protocol will choose communication instants such that they coincide with sampling instants. At least $N$ sampling instants will need to elapse between two consecutive communications, where $N$ is a choosable parameter. This parameter is finite and a part of the communication protocol, which implies that it is known by all interacting agents. The choice of $N$ directly influences the maximum observation error and resulting rate. How exactly one might choose $N$ and how it influences the error will be discussed in the next section.

To properly describe the communication instants, we will need several quantities. The indexes $j$ of the communication instants are inherently known by all agents. The quantity $\bar{j}$ refers to the index of the last instant of communication (initially, $\bar{j}=0$ ). This quantity is always known by the sampler (because it knows how many communication instants it defined), the coder (because it knows how many messages it sent), as well as the decoder (because it knows how many messages it received). Finally, the sampler and coder interact to update the knowledge of the estimate $\hat{x}(t)$ at the coder side.

Procedure 1:

The Sampler $\mathscr{S}$ : At each sampling instant $t_{k} \geq s_{\bar{j}}+N \bar{t}$, the sampler computes $\hat{x}_{c}\left(t_{k}\right)$ and then verifies whether the following condition is satisfied

$$
\left\|y\left(t_{k}\right)-\hat{x}_{c}\left(t_{k}\right)\right\|_{2} \leq \varepsilon_{x}+2\left|\bar{u}_{x}\right| N \bar{t}+2 \delta_{x} N \bar{t}-2\left|\bar{u}_{x}\right| \bar{t}-2 \delta_{x} \bar{t} .
$$

If the condition is not satisfied, a message must be sent to provide a new estimate. The sampler thus sets $s_{\bar{j}}=t_{k}$ and $\bar{j}$ increases by 1 .

The Alphabet Function $\mathscr{A}$ : If $t_{k}=s_{\bar{j}}$, the alphabet agent builds a covering of the set $I_{\bar{j}}$, where $I_{\bar{j}}$ is defined as

$$
\begin{aligned}
I_{\bar{j}}:= & \left\{x \in \mathbb{R}^{2} \mid\left\|x-\hat{x}_{c}\left(s_{\bar{j}-1}\right)\right\|_{2} \leq \varepsilon_{x}+2\left(\left|\bar{u}_{x}\right|+\delta_{x}\right) N \bar{t},\right. \\
& \left.\left\|x-\hat{x}_{c}\left(s_{\bar{j}-1}\right)\right\|_{2} \geq \varepsilon_{x}+2\left(\left|\bar{u}_{x}\right|+\delta_{x}\right) N \bar{t}-2\left(\left|\bar{u}_{x}\right|+\delta_{x}\right) \bar{t}\right\},
\end{aligned}
$$

with disks of radius $\varepsilon_{x}$. The disks in this covering are numbered from 1 till $l_{\bar{j}}^{x}$. The coder also divides $S^{1}$ into intervals of size $2 \varepsilon_{\theta}$. The these intervals are numbered from 1 till $l_{\bar{j}}^{\theta}$. The alphabet function then returns $l_{\bar{j}}=l_{\bar{j}}^{x} l_{\bar{j}}^{\theta}$.

The Coder $\mathscr{C}$ : At the communication instants, the coder function finds the index of the ball in the covering made by the alphabet whose center is the closest to $x\left(s_{\bar{j}}\right)$. The coder also finds the index of the interval in the covering of $S^{1}$ which contains $\theta\left(s_{\bar{j}}\right)$. It then sends both indexes to the decoder. The coder also updates the local estimates $\hat{x}_{c}\left(s_{\bar{j}}\right)$ and $\hat{\theta}_{c}\left(s_{\bar{j}}\right)$ by setting them to be equal to the center of the ball in $I_{j}$.

The Decoder $\mathscr{D}:$ In the absence of messages, the decoder computes $\hat{x}(t)$ and $\hat{\theta}(t)$ as solutions of (12) with $\hat{x}\left(t_{\bar{j}}\right)$ and $\hat{\theta}\left(t_{\bar{j}}\right)$ as an initial conditions. If a message is received, the decoder uses the center of the disk whose index it received as $\hat{x}\left(t_{\bar{j}}\right)$ and the center of the interval whose index it received as $\hat{\theta}\left(t_{\bar{j}}\right)$.
The alphabet is based on the following idea. As was previously mentioned, in the absence of messages, new estimates are obtained at the decoder side by solving (12). After receiving a message, the state of the system $x(t)$ is contained in a ball of a radius $\varepsilon_{x}$ whose center is the estimate $\hat{x}(t)$. In the absence of any messages, the distance between the state and the estimate increases/decreases (both cases are possible). The distance between the state and estimate evolves due to two factors: first of all, the unknown state perturbation $d_{x}(t)$ and $d_{\theta}(t)$ increase it continuously (but no more than $\delta_{x}$ every second). Secondly, the distance set is increased/decreased by the action of the system dynamics. Given that the communication intervals are chosen to be finite (i.e., $N<\infty$ ), the distance remains finite in between communications. Supposing that the set $I_{j}$ is defined in such a way that it always contains the current state at the communication instants, then by covering this set with balls of radius $\varepsilon_{x}$, the centers of the balls of which cover $I_{j}$ form an alphabet to communicate estimates $\hat{x}$.

In this case, the set $I_{j}$, which is simply a ring centered around the previous estimate, can be covered by a finite number of balls of size $\varepsilon_{x}>0$. The balls in the covering can be indexed from 1 till $l_{\max }<\infty$. To produce such a covering, the only information needed is the initial ball and the different upper bounds on the uncertainties/errors, which implies that both the coder as well as the decoder can build the set. To transmit a new estimate of $x$, one can simply send the index of one of the balls whose center then serves as a new estimate with a precision that will depend on $\varepsilon_{x}$. The cost of communicating in that fashion is dependent on how many balls of size $\varepsilon_{x}$ are required to cover $I_{j}$. We finish the current section with several remarks on the different features of the proposed scheme.

Remark 2: - The copies of the estimates at the sensor side $\hat{x}_{c}(t)$ and $\hat{\theta}_{c}(t)$ are either updated by the sampler if no message is sent, or by the coder if a message is sent.

- The coordinates of the centers of the balls used in the covering are always relative to the previous estimates. By communicating in relative fashion, it is possible to keep the size of the messages limited. Note that since $\hat{x}_{c}(t)=\hat{x}(t)$, both agents can build this set according to its definition (15).

- The alphabet procedure is easy from a computational point of view since it consists of covering one set which always has the same shape except the whole set is shifted by a certain vector from the origin and another set which is simply $S^{1}$. The first of these sets is centered around the previous estimate, both the coder and decoder can build a covering for it and thus have access to the alphabet.

- The computational requirement on the decoder are relatively low since an exact solution (13) exists to (12).

\section{RATE AND ERRORS}

With the observer and its agents fully introduced, we are in a position to determine a bound on the communication rate resulting from the observer. This quantity is related to the observation error, for which we also provide a theoretical bound. The first result provides a closed-form expression of the upper bound of the total estimation error that indicates the proportionality of the different parameters/errors.

Proposition 1: The observer described in Procedure 1 ensures that $\eta \leq \varepsilon_{x}+2\left(\left|\bar{u}_{x}\right|+\delta_{x}\right) N \bar{t}$, where $\eta$ is defined in (11).

The proof of all results have been omitted due to space limitations. They are available in the appendices of Chapter 6 of [26].

Remark 3: The use of Proposition 1 is straightforward. Based on the velocities $\bar{u}_{x}$ and $\bar{u}_{\theta}$, as well as the bounds on the perturbations 
$\delta_{x}$ and $\delta_{\theta}$, the user of the communication scheme has an expression that links the minimum number of sampling intervals between communications, the discretization error $\varepsilon_{x}$ and the total error. Depending on the maximum tolerable observation error, it is thus simple to find $N$ such that the error will not exceed that bound.

The next result of this section aims to provide an upper bound on $R$ for the designed communication scheme.

Theorem 1: The observer described in Procedure 1 with $N>0$ results in a communication rate $R$ such that

$R \leq \frac{1}{N \bar{t}}\left\lceil\log _{2}\left\lceil\frac{\pi}{\varepsilon_{\theta}}\right\rceil\left\lceil\frac{2\left(\varepsilon_{x}+2\left|\bar{u}_{x}\right| N \bar{t}+2 \delta_{x} N \bar{t}\right) \pi}{\sqrt{2} \varepsilon_{x}}\right\rceil\left\lceil\frac{2\left|\bar{u}_{x}\right| \bar{t}+2 \delta_{x} \bar{t}}{\sqrt{2} \varepsilon_{x}}\right\rceil\right\rceil$.

Note that in the proof of Theorem 1 a covering procedure is presented for $I_{j}$. This covering procedure is very conservative. There exist coverings of rings with disks that require fewer disks but the objective of this theorem is simply to provide a theoretical upper bound on the required communication rate. In practice, the communication protocol requires a much lower rate than the theoretical bound, as will be shown through experiments in the next section.

\section{EXPERIMENTS}

In order to test the observer, experiments were run in a lab on a Turtlebot $2^{1}$, which is a unicycle-type robot. The Turtlebot is equipped with a netbook, which utilizes ROS (Robot Operating System $^{2}$ ). Four sets of experiments were run in total, each with the objective to test one particular configuration. All experiments were run with the following setting for the velocities $\left(\bar{u}_{x}, \bar{u}_{\theta}\right)=$ $(0.1[\mathrm{~m} / \mathrm{s}],-0.2[\mathrm{rad} / \mathrm{s}])$. The precision of the estimates is $\left(\varepsilon_{x}, \varepsilon_{\theta}\right)=$ $(0.01[\mathrm{~m}], 0.01[\mathrm{rad}])$. The four sets of experiments are:

1) No perturbations: The first set of experiments consists of steering the robot with the constant velocities without any additional perturbations;

2) Small angular velocity perturbations: The second set of experiments revolves around testing a configuration with perturbations only in the angular velocity;

3) Larger perturbations: The third set of experiments involves large perturbations, both in the linear velocity and in the angular velocity;

4) Larger perturbations, larger observation error: The fourth and final set of experiments involves the same perturbations as the third set of experiments except $N$ is now chosen larger.

A video of the experiments (as well as Gazebo simulations, which are not presented in this work, due to space limitations) is available at the following URL: https://www - youtube.com/watch? $\mathrm{V}=\mathrm{z} \times 3 \mathrm{Mckyj} 4 \mathrm{EM}$.

\section{A. First Experiment - No Perturbations}

In this experiment, the dynamics of the robot are assumed to be unperturbed (i.e., $\delta_{x}=\delta_{\theta}=0$ ). The sampling time for the robot is $\bar{t}=0.5 \mathrm{~s}$. We choose $N=2$ (implying that a communication could potential occur every second). The following bounds are then obtained on $\eta$ and $R$ by applying Proposition 1 and Theorem $1: \eta \leq 0.21[\mathrm{~m}], R \leq 17$ [bits/s]. Several experiments are run, each consisting of 120 seconds. A typical trajectory in the $x_{1}, x_{2}$-plane is depicted on Fig. 2 while the observation error for the same run is depicted in Fig. 3.

\footnotetext{
${ }^{1}$ See https://www.turtlebot.com/turtlebot2/ for more information.

${ }^{2}$ ROS is an open-source operating system to control robots, more information available at https://www.ros.org/.
}

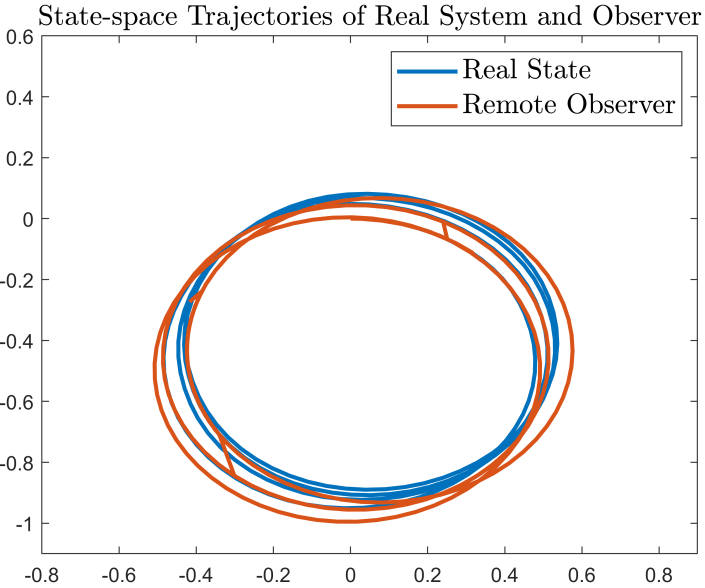

Fig. 2. Figure depicting the state-space trajectory of the robot (blue) and the remote estimate (orange) for the unperturbed case.

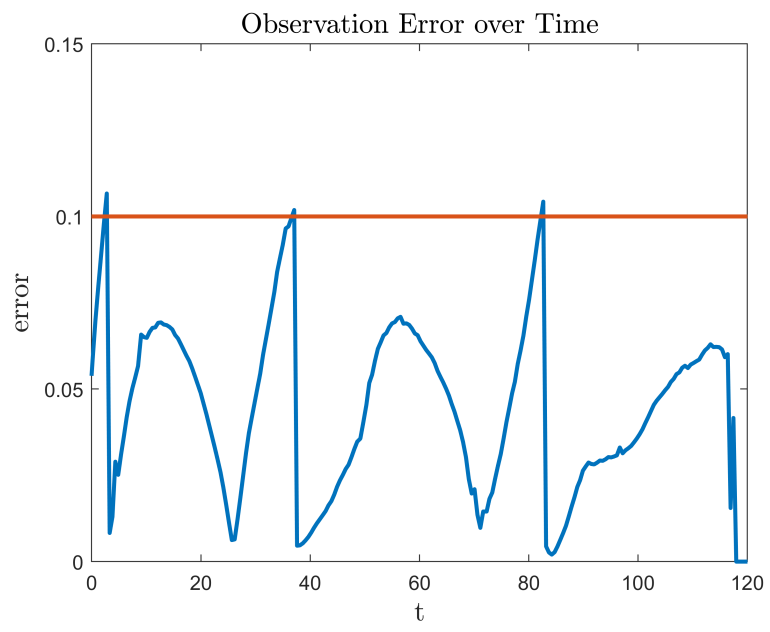

Fig. 3. Figure depicting the observation error (blue), together with the triggering condition (orange) for the unperturbed case.

As can be seen from the figures, even in the unperturbed case, several communications are required. This is due to internal frictions of the robot, imperfect actuation, frictions with the floor, ... all of which are not accounted for in the model. On average, over 10 different trials, the number of communications is 4.1 per 120s. This is still much lower than the upper bound on the theoretical rate predicted, 120 communications. The resulting rate is $17 \times 4.1 / 120=0.5805$ [bits/s], which is 34 times lower than the upper bound on the theoretical rate, which proves the effectiveness of the event-triggered protocol, as well as some conservatism in the theoretical error bounds. In terms of observation error, the error remains much below the maximum observation bound.

\section{B. Second Experiment - Small Angular Velocity Perturbations}

In this experiment, the dynamics of the robot are assumed to only be perturbed in angular velocity. The sampling time for the robot is $\bar{t}=0.5 \mathrm{~s}$. We use $\delta_{x}=0$ and $\delta_{\theta}=0.05$ (implying up to $25 \%$ variation in the angular velocity). We again choose $N=2$ (implying that a communication could potential occur every second). The following bounds are then obtained on $\eta$ and $R$ by applying Proposition 1 and Theorem 1: $\eta \leq 0.21[\mathrm{~m}], R \leq 17$ [bits/s]. Note that since only $\delta_{\theta}$ changed from the first experiments, the bounds are identical. Several experiments are run, each consisting of 120 seconds. A 
typical trajectory in the $x_{1}, x_{2}$-plane is depicted on Figure 4 while the observation error for the same run is depicted in Figure 5.

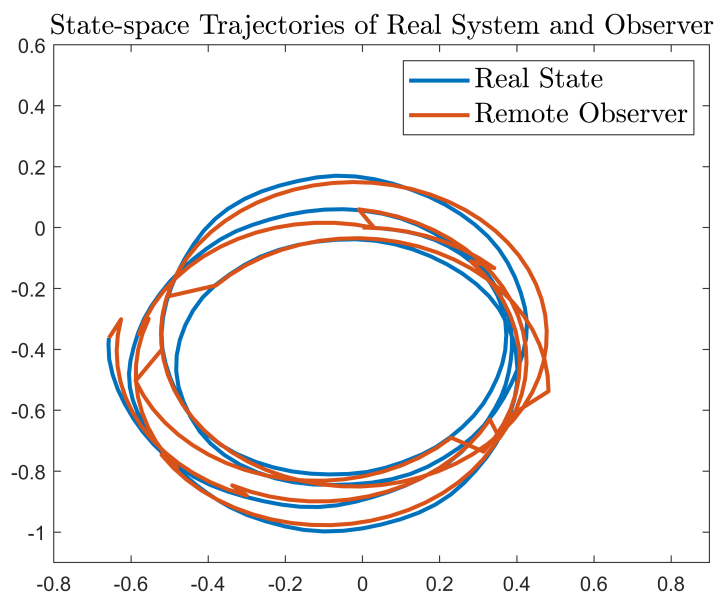

Fig. 4. Figure depicting the state-space trajectory of the robot (blue) and the remote estimate (orange) for the case with angular velocity perturbations.

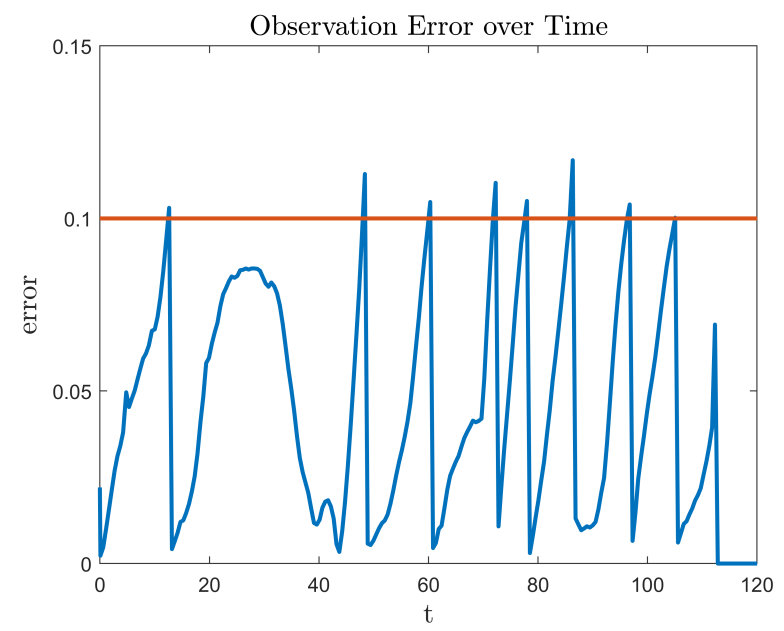

Fig. 5. Figure depicting the observation error (blue), together with the triggering condition (orange) for the case with angular velocity perturbations.

This time, the state-space trajectories of the system deviate much more compared to the unperturbed case. The effects of the perturbation on the angular velocity can clearly be seen. Over 10 experiments, an average of 10.5 communications are necessary, which is much lower than the upper bound on the theoretical rate of 120. The resulting rate is $17 \times 10.5 / 120=1.3125$ [bits $/ \mathrm{s}]$. This again validates the effectiveness of the event-triggered communication scheme.

\section{Third Experiment - Large Perturbations}

In this experiment, the dynamics of the robot are assumed to be perturbed by large perturbations. We use $\delta_{x}=0.2$ and $\delta_{\theta}=$ 0.2 . This time, the sampling interval is assumed to be $\bar{t}=0.1$. We choose $N=3$ (implying that a communication could potential occur every 0.3 seconds). The following bounds are then obtained on $\eta$ and $R$ by applying Proposition 1 and Theorem 1: $\eta \leq 0.19$ [m], $R \leq 53.33$ [bits/s]. Several experiments are run, each consisting of 120 seconds. A typical trajectory in the $x_{1}, x_{2}$-plane is depicted on Figure 6.

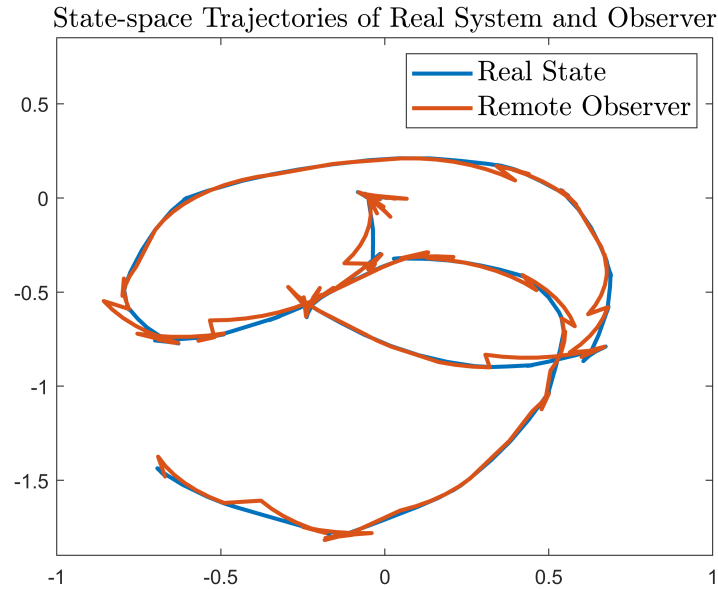

Fig. 6. Figure depicting the state-space trajectory of the robot (blue) and the remote estimate (orange) for the large perturbations case.

The effects of these large perturbations are immediately seen on the state-space trajectories of the robot. Many more communications are therefore required: on average 53.6 over 120 seconds. This results in an effective communication rate of $16 \times 53.6 / 120=$ 7.1467 [bits/s]. This is again much below the upper bound on the theoretical rate.

\section{Fourth Experiment - Large Perturbation Large Observation Error}

For the final round of experiments, the same setting as the previous experiments is assumed, except $N=4$ (implying that a communication could potential occur every 0.4 seconds). The following bounds are then obtained on $\eta$ and $R$ by applying Proposition 1 and Theorem 1: $\eta \leq 0.25$ [m], $R \leq 40$ [bits/s]. The different choice of $N$ thus immediately impacts both the maximum error, which becomes larger and the upper bound on the theoretical rate, which becomes smaller. Several experiments are run, each consisting of 120 seconds. A typical trajectory in the $x_{1}, x_{2}$-plane is depicted on Figure 7.

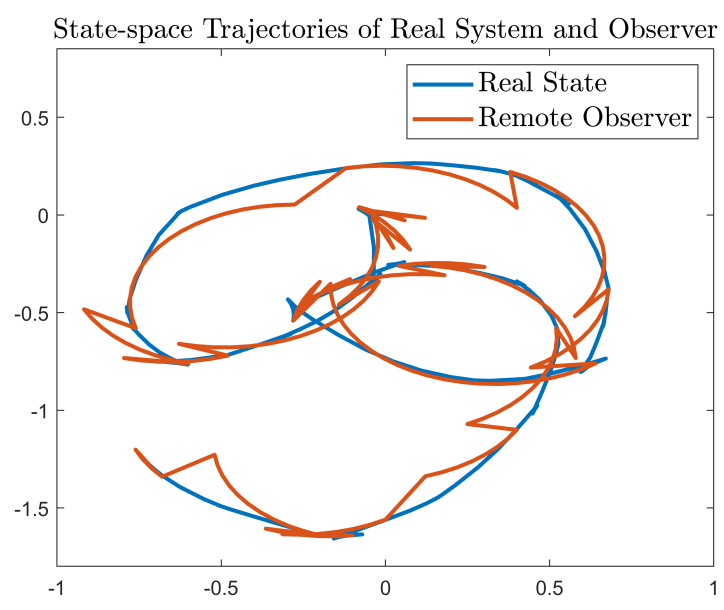

Fig. 7. Figure depicting the state-space trajectory of the robot (blue) and the remote estimate (orange) for the large perturbations case with larger observation error.

The experiments confirm the same effect as the theoretical bounds: as can be seen in Figure 7, the maximum distance between the observed trajectory and the actual state-space trajectory is 
larger than in the previous experiment (see Figure 6). In terms of the number of communications, the average now sits at 24.2 communications per 120 seconds, which implies an effective rate of $16 \times 24.2 / 120=3.2267$ [bits/s], almost twice as low as in the previous experiment. This confirms the fact that there is a tradeoff between precision and rate that can be tuned through $N$.

\section{CONCLUSION}

In this document, we presented an event-triggered, data rate constrained observer for unicycle-type robots with constant velocities and time-varying perturbations. After posing the problem statement, the design of the agents that form the communication protocol was developed. Two theoretical results were presented. First, a proposition which links the minimum time interval between two consecutive communications and the maximum error. Secondly, a theorem that upper bounds the communication rate resulting from the communication protocol. The effectiveness of the proposed communication scheme was experimentally validated on Turtlebots.

We conclude with the following remarks on the communication protocol:

1) The communication protocol is very efficient at producing precise estimates at a remote distance;

2) The required communication rate is much lower than the upper bound on the theoretical rate, which is due both to the usage of an event-triggered communication protocol, as well as conservatism in the error bounds;

3) It is possible to exchange more precision for a higher communication rate and vice-versa, by tuning the parameter $N$;

4) Although a proportion of the transmitted bits is used to transmit $\hat{\theta}$, which is not included in the observation error, it is an essential part of the dynamics of the robot and hence plays a crucial role in increasing the time between two subsequent communications (by improving the quality of the remote estimate).

Further research on this topic includes configurations with piecewise constant input, tracking of the full state instead of only the position, improving the covering procedure to improve the theoretical bounds, and studying the fundamental minimum requires capacity to observe unicycle-type robots.

\section{REFERENCES}

[1] Karl-Erik Åarzén. A simple event-based PID controller. IFAC Proceedings Volumes, 32(2):8687-8692, 1999.

[2] Boris R. Andrievsky, Alexey S. Matveev, and Alexander L. Fradkov. Control and Estimation under Information Constraints: Toward a Unified Theory of Control, Computation and Communications. Automation and Remote Control, 71(4):572-633, 2010.

[3] Karl Johan Åström and Bo Bernhardsson. Comparison of periodic and event based sampling for first-order stochastic systems. In Proceedings of the 14th IFAC World Congress, pages 5006-5011, Beijing, 1999.

[4] John Baillieul and Panos J Antsaklis. Control and Communication Challenges in Networked Real-Time Systems. Proceedings of the IEEE, 95(1):9-28, 2007.

[5] Nicola Elia and Sanjoy K. Mitter. Stabilization of linear systems with limited information. IEEE Transactions on Automatic Control, 46(9):1384-1400, 2001.

[6] Willem Jan Evers and Henk Nijmeijer. Practical stabilization of a mobile robot using saturated control. In Proceedings of the 45th IEEE Conference on Decision and Control, pages 2394-2399, San Diego, 2006.

[7] Duo Han, Yilin Mo, Junfeng Wu, Sean Weerakkody, Bruno Sinopoli, and Ling Shi. Stochastic event-triggered sensor schedule for remote state estimation. IEEE Transactions on Automatic Control, 60(10):2661-2675, 2015.
[8] W. P.M.H. Heemels, Karl Henrik Johansson, and P Tabuada. An Introduction to Event-triggered and Self-triggered Control. In Proceedings of the 51st IEEE Conference on Decision and Control, pages 32703285, Maui, 2012.

[9] J.P. Hespanha, P. Naghshtabrizi, and Yonggang Xu. A Survey of Recent Results in Networked Control Systems. Proceedings of the IEEE, 95(1):138-162, 2007.

[10] Laurentiu Hetel, Christophe Fiter, Hassan Omran, Alexandre Seuret, Jean-pierre Richard, and Silviu Iulian Niculescu. Recent developments on the stability of systems with aperiodic sampling : An overview. Automatica, 76:309-335, 2017.

[11] Zhong Ping Jiang and Henk Nijmeijer. Tracking Control of Mobile Robots: A Case Study in Backstepping. Automatica, 33(7):1393-1399, 1997.

[12] Christoph Kawan. Invariance Entropy for Deterministic Control Systems An Introduction. Springer Cham Heidelberg New York Dordrecht London, 2013.

[13] Christoph Kawan. Exponential state estimation, entropy and Lyapunov exponents. Systems and Control Letters, 113:78-85, 2018.

[14] D. Kostic, S. Adinandra, J. Caarls, N. Van De Wouw, and H. Nijmeijer. Collision-free tracking control of unicycle mobile robots. In Proceedings of the 48th IEEE Conference on Decision and Control held jointly with the 2009 28th Chinese Control Conference, pages 5667-5672, Shanghai, 2009.

[15] Daniel Liberzon and Joao. P. Hespanha. Stabilization of nonlinear systems with limited information feedback. IEEE Transactions on Automatic Control, 50(6):910-915, 2005.

[16] Daniel Liberzon and Sayan Mitra. Entropy and minimal data rates for state estimation and model detection. In Proceedings of the 16' International Conference on Hybrid Systems: Computation and Control, pages 247-256, Vienna, 2016.

[17] Alexey S. Matveev and Alexander Yu. Pogromsky. Observation of nonlinear systems via finite capacity channels: Constructive data rate limits. Automatica, 70:217-229, 2016.

[18] Alexey S. Matveev and Alexander Yu Pogromsky. Observation of nonlinear systems via finite capacity channels, Part II: Restoration entropy and its estimates. Automatica, 103:189-199, 2019.

[19] Alexey S. Matveev and Andrey V. Savkin. Estimation and Control over Communication Networks. Birkhäuser Boston Basel Berlin, 2009.

[20] Michael Muehlebach and Sebastian Trimpe. Distributed event-based state estimation for networked systems: An LMI approach. IEEE Transactions on Automatic Control, 63(1):269-276, 2018.

[21] Girish N. Nair, Robin J. Evans, Iven M.Y. Mareels, and William Moran. Topological feedback entropy and nonlinear stabilization. IEEE Transactions on Automatic Control, 49(9):1585-1597, 2004.

[22] Claude E. Shannon. A Mathematical Theory of Communication. Bell System Technical Journal, 27(3):379-423, 1948.

[23] Hussein Sibai and Sayan Mitra. Optimal Data Rate for State Estimation of Switched Nonlinear Systems. In Proceedings of the 17' International Conference on Hybrid Systems: Computation and Control, pages 71-80, Pittsburgh, 2017.

[24] Hussein Sibai and Sayan Mitra. State Estimation of Dynamical Systems with Unknown Inputs: Entropy and Bit Rates. In Proceedings of the 18' International Conference on Hybrid Systems: Computation and Control, pages 217-226, Porto, 2018.

[25] Sebastian Trimpe. Event-based state estimation: An emulationbased approach. IET Control Theory and Applications, 11(11):1684-1693, 2017.

[26] Quentin Voortman. Networked Control and Estimation Under Restrictions on Channel Capacity. PhD thesis, Eindhoven University of Technology and Ecole Centrale de Lille, 2021.

[27] Quentin Voortman, Denis Efimov, Alexander Yu Pogromsky, Jeanpierre Richard, and Henk Nijmeijer. Synchronization of Perturbed Linear Systems with Data-Rate Constraints. In Proceedings of the 59th IEEE Conference on Decision and Control, Jeju Island, 2020.

[28] Quentin Voortman, Alexander Yu Pogromsky, Alexey S Matveev, and Henk Nijmeijer. Data-Rate Constrained Observers of Nonlinear Systems. Entropy, 21(282):1-29, 2019.

[29] Wing Shing Wong and Roger W. Brockett. Systems with finite communication bandwidth constraints - Part I: State estimation problems. IEEE Transactions on Automatic Control, 42(9):1294-1299, 1997.

[30] Meng Xia, Vijay Gupta, and Panos J Antsaklis. Networked State Estimation Over a Shared Communication Medium. IEEE Transactions on Automatic Control, 62(4):1729-1741, 2017. 